\title{
NEEDLE-FREE INJECTION SYSTEM
}

\author{
M. V. DUKARE ${ }^{*}$, R. B. SAUDAGAR ${ }^{2}$
}

1*Department of Quality Assurance Techniques, R. G. Sapkal College of Pharmacy, Anjaneri, Nashik 422213, Maharashtra, India, ${ }^{2}$ Department of Pharmaceutical Chemistry, R. G. Sapkal College of Pharmacy, Anjaneri, Nashik 422213, Maharashtra, India Email: monidukare1@gmail.com

Received: 25 Dec 2017, Revised and Accepted: 05 Feb 2018

\begin{abstract}
Needle free injection system are to introduce the various medicines into patients without piercing the skin with a conventional needle. Needle-free technology offers the many benefit of reducing patient concern about the use of needle. Needle free injection is the very effective injections a wide range of drugs and bioequivalent to syringe and needle. It results in less pain, and is strongly preferred by patients. Additional benefits include very fast injection compared with conventional needles and no needle disposal issues. Not only benefit of the pharmaceutical industry to the increasing product sales, it has the added potential to increase compliance with dosage regimens and improved outcomes. Today, they are a steadily developing technology that promises to make the administration of medicine more efficient and less painful.
\end{abstract}

Keywords: Needle free injection, Needle free devices, Needle free technology, Drug administration and drug delivery system

(C) 2018 The Authors. Published by Innovare Academic Sciences Pvt Ltd. This is an open access article under the CC BY license (http://creativecommons.org/licenses/by/4.0/) DOI: http://dx.doi.org/10.22159/ijcpr.2018v10i2.25885

\section{INTRODUCTION}

The needle free is used to describe the an extensive range of drug delivery technologies, which consists of technologies that do not have a needle but make use of electrophoresis to drive drugs through the skin, technologies that use one or more very small needles, but needles nevertheless. Needle free devices are taken to the form of power sprays, edible products, inhalers, and skin patches. Devices are available in reusable and disposable forms, for home or physicians office use, and also in versions for multiple patients and institutional uses [1].

\section{Disadvantageous $[1,2]$}

1. The risk of cross-contamination from needle stick injury

2. Under or overdosing which results in poor injection technique in patients

3. Needle phobia

4. Injection site pain

5. Poor compliance resulting in long-term worsening of conditions
6. The Increased costs due to the patients visiting the hospitals for injections

\section{History}

The first syringes were the first developed by a French surgeon, Charles Gabriel pravaz, in 1853, hypodermic there is a minor development in syringes is the technology has been remained unchanged for last $150 \mathrm{y}$. Needle-free systems were the first described by marshalllockhart in 1936 in his patent jet injection. Then in the early 1940's higson and others developed high pressure "guns"using a fine jet of liquid to pierce the skin and deposit the drug in underlying tissue [3]

\section{Structure of human skin [4-7]}

The knowledge of the structure in the skin is essential to the successful administration of drugs through the needle free injection systems as these drugs are administered to the skin.

Human skin is generally made of two layers [1].

- Epidermis

- Dermis.

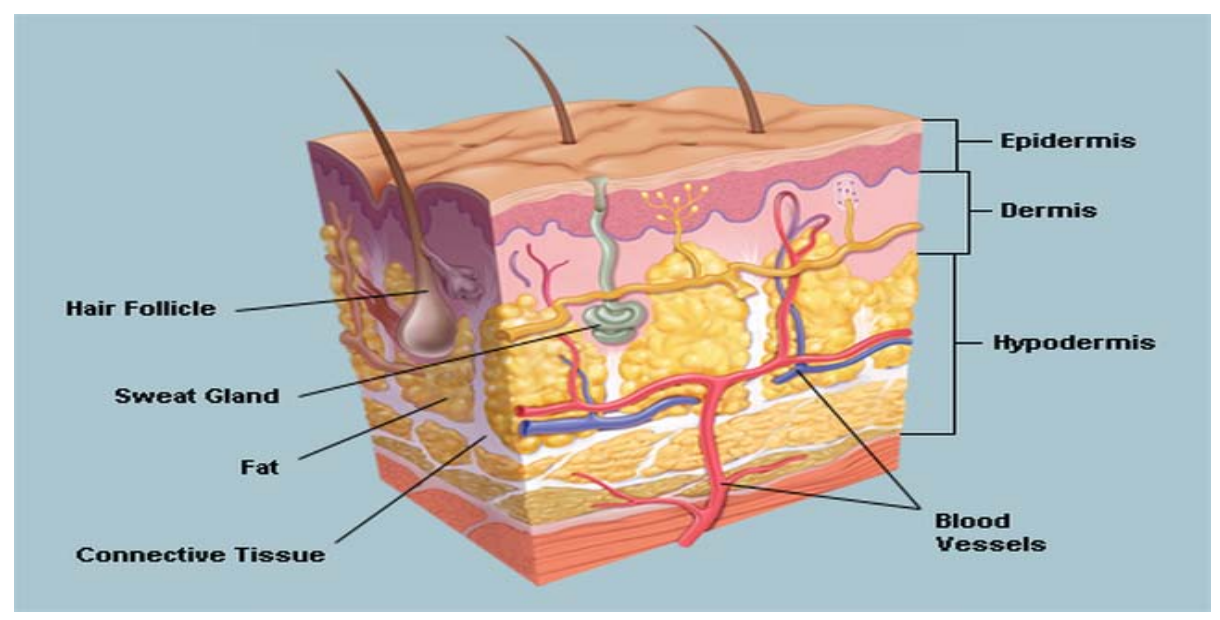

Fig. 1: Layer of skin 


\section{The epidermis}

This is a stratified squamous epithelium layer i.e. are composed primarily of two types of cells: keratinocytes and dendritic cells. The Epidermis layer are harbour a number of other cells such as melanocytes, Langerhans cells and Merkel cells. But the keratinocytes cells types comprises the majority of the cells by far. The layers of epithelium are

\section{Stratum germinativum}

It contains column-shaped keratinocytes that attach to the basement membrane zone with their long axis perpendicular to the dermis.

\section{Stratum spinosum}

Its thickness varies from 5-10 cells. Intercellular spaces between spinous cells are bridged by abundant desmosomes (adhering spot) that promote coupling between cells of the epidermis and provide resistance to physical stresses.

\section{Stratum granulosum}

It contains living cells, these are responsible for further synthesis and modification of proteins involved in keratinization. It is 1-3 cells layer in thickness.

\section{Stratum corneum}

The corneocytes are rich in protein and low in lipid content (hydrophilic nature) are surrounded by a continuous extracellular lipid matrix.

\section{Malpighian layer}

The layer whose protoplasm has not yet change into horny material.

\section{The dermal-epidermal}

It acts as a support for the epidermis, establishes cell polarity and direction of growth, directs the organization of the cytoskeleton in basal cells, provides developmental signals and function as a semipermeable barrier between layers.

\section{The dermis}

It is on an integrated system of fibrous, filamentous and amorphous connective tissue that accommodates stimulusinduced entry by nerve, vascular-networks, appendages, fibroblasts, mast cells. Its thickness ranges from $2000-3000 \mu \mathrm{m}$ The principal component of the dermis is collagen and represents $70 \%$ of the skin's dry weight.

\section{Sub-cutaneous (Connective Tissue)}

The subcutaneous tissue or hypodermis is not actually considered a true part of the structured connective tissue which is composed of loose textured, white, fibrous connective tissue containing blood and lymph vessels, secretary pores of the sweat gland and cutaneous nerves. Most investigators consider drug permeating through the skin enter the circulatory system before reaching the hypodermis, although the fatty tissue could serve as a depot of the drug.

\section{Advantageous [8]}

- Prevent skin puncture hazards and its destruction; also does not cause the problem of bleeding or bruising and minimal skin response [8].

- Imparts fast drug delivery and better reproducibility as compared to invasive drug delivery systems and hence enhance bioavailability when compared with invasive drug delivery systems.

- Better drug stability during storage as it is delivered in dry powder form especially for water sensitive drugs.

- Avoids problems of reconstitution and any effect of shearing.

- Elimination of needle phobia.

- Self-administration is feasible with needle free injections.

- Improves immune response to vaccines. Immunization of influenza, tetanus, typhoid, diphtheria, pertussis, and hepatitis A vaccines can be delivered by needle free injections.

- Bio-equivalence has been demonstrated enabling the development of generic drug proteins.

- A good dose response with increased drug doses.

\section{Disadvantages [8]}

1. The method is complex and expensive.

2. All systems are not fitted into one size.

3. Need for personnel training and maintenance.

4. It is not applicable for the Intravenous route.

\section{Types of needle free injection}

Needle-free injection drug delivery systems are classified as follows [9]

1. Powder injections

2. Liquid injections

3. Depot or Projectile Injection.

\section{System type 1-powder injections}

\section{Design of powder injection systems}

These injections consist of a chamber filled with solid drug content and a nozzle for firing drug particles into the skin by utilizing the power source which typically is compressed gas. The injection has a diaphragm (a few microns thick) on either side of the chamber to cover the drug chamber [9].

\section{Mechanism of powder injection}

- Particles exist from the nozzle along with a gas stream.

- Particles impinge the skin surface leading to the formation of a hole into the skin with the progression of the injection.

- Drug particles get deposited in a spherical pattern at the end of the hole and penetrate across the stratum corneum.

- After their penetration into the skin, drug particles get distributed completely into the stratum corneum and the viable epidermis.

Powder injection is accomplished by a light gas gun. It provides the required particle velocity by use of an accelerating piston which accelerates and carries particles with it. Particles leave piston surface by means of a deacceleration mechanism which slows down the piston. This leads to ejection of particles that act on the target tissue area [10].

(c)

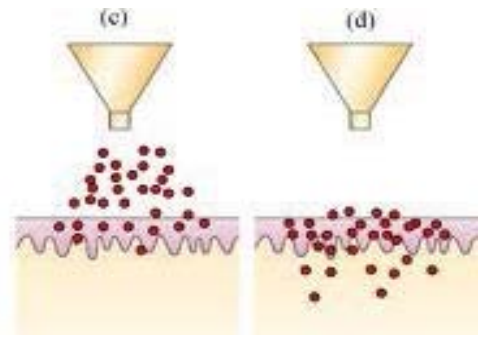

Fig. 2: Mechanism of a powder injection [10] 


\section{Ideal characteristics of powder particles [11]}

- In case of powder, injections is the drug particle size distribution, quality, its physical and chemical stability are extremely important.

- Powder in an injection may be a whole drug or a formulation containing drug with excipients for dilution purposes or to stabilize the product. Therefore, drug and other excipients must be compatible with each other.

- Particle size are plays an important role in penetration into the stratum corneum; hence it should remain uniform throughout usage and storage.

- The particles must be robust enough to survive the highly energetic gas jet within the device as well as ballistic impact with the skin. As the particles strike the skin at a high velocity then they must be strong. The particles is clocked as fast as 900 meters per second, with 400 to 600 meters per second being the more typical range.

- In order to exert required effects in the body after being absorbed into systemic circulation, the drug particles should have proper diffusion within the skin.

- For skin penetration at a high velocity, the powders must have particle densities of about $1 \mathrm{~g} / \mathrm{cc}$ and mean diameter greater than 20 $\mu \mathrm{m}[11]$.

\section{Advantages of powder injections $[10,12]$}

- A small volume of material is shot through the skin as a drug, is in powder form instead of liquid form, hence injection is painless.

- The therapeutic agent will be more stable and then is no need of cold storage.

- The sustained release effect or drug performance achieved by using bio erodible carriers, slowly dissolving excipients specific, less soluble salts or dissolution aids.

(a)

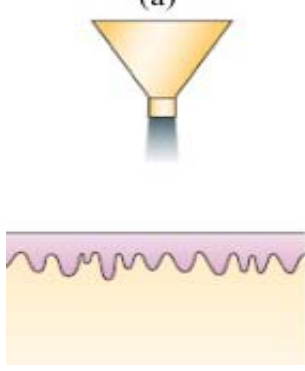

(b)

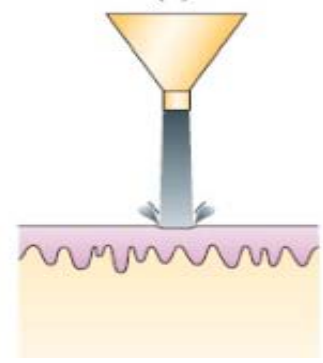

- Protein drugs are very potent, and then suitable for powder needle free injection systems.

\section{System type 2-liquid injections}

The basic principle of injection is "if a high enough pressure is generated by a fluid in intimate contact with the skin, then the liquid will punch a hole into the skin and be delivered into the tissues in and under the skin." Although the same principle is applied as in powder, there is a difference in the actual design and operation of the powder injection devices. These systems use gas or spring, pistons, drug-loaded compartments and nozzles. Typically, the nozzle has an orifice size of about 150 to $300 \mu \mathrm{m}$ [12]

\section{Mechanism of liquid injections}

Impact of a piston on a liquid reservoir in the nozzle increases the pressure, which shoots the jet out of the nozzle at high velocity the velocity is $>100 \mathrm{~m} / \mathrm{s}$

The effect the jet on the skin surface starts the formation of a hole in the skin through erosion, fracture, or other skin failure mechanisms.

Further impingement of the jet increases the depth of the hole in the skin. If the volumetric rate of hole formation is less than the volumetric rate of jet impinging on the skin, then some of the liquid splashes back towards the injector.

The accumulation of the liquid in the hole occurs because of a deeper hole in the skin which slows down the incoming jet. Then further development of a hole is stopped. The dimensions of the hole are established very early in the process from the time of impact. Stagnation of the jet at the end of the hole disperses the liquid into the skin in a near-spherical shape [10].

\section{System type 3-depot or projectile injections}

These systems are the designed for administration of a drug into muscles. They are creating a store of the drug into muscles that is released continuously over a desired time period [12].

Fig. 3: The mechanism of a liquid injection [10]

(c)

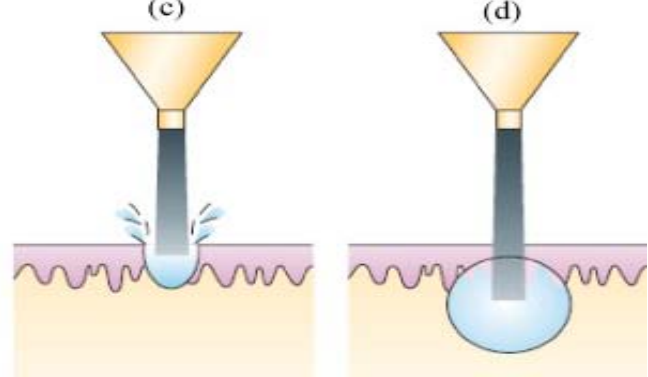

Table 1: Market product of liquid-based needle free injection [13-20]

\begin{tabular}{lll}
\hline Product name & Company & Types of system \\
\hline Intraject & Weston medical & Liquid-based needle free injection \\
Medijector vision & Antares Pharma Inc. & Liquid-based needle free injection \\
Penjet & Penjet corporation & Liquid-based needle free injection \\
Med-E-Jet & Evans enterprise & Liquid-based needle free injection \\
Advantaget & Advantage health services & Liquid-based needle free injection \\
Gentlejet & Health for personal care & Liquid-based needle free injection \\
J-tip & National medical products, inc & Liquid-based needle free injection \\
Injex & Equidyne Systems, Inc & Liquid-based needle free injection \\
Powderject system & Powder ject pharmaceuticals & Powder-based needle free injection \\
DepixolDepo injection & Lundbeck Limited & Depot based needle free injection \\
\hline
\end{tabular}

\section{Injection methods $[21,22]$}

Subcutaneous injections system in the small pigs should be given by pulling the loose skin in the elbow or flank area. This technique is called the tenting. In sows, the area just behind the ear is an acceptable sight for SQ injection. Intramuscular injection is conventionally administered in the neck just behind the ear. IM injection anywhere else is not acceptable because it will compromise pork safety and it should never be injected into the ham muscles and loin.

Types of needle-free injection systems $[21,22]$

Needle-free injection systems are not a new development. The earliest systems were developed in the 1930s and used in a wide 
variety of medical areas over the years. 4 Through innovation and technology, there have been modifications and variations that allow for needle-free injection systems to be more widely available and effective to consumers.

- Spring load jet injector

- Battery powdered jet injector

- Gas powdered jet injector

\section{Spring-load jet injector [21, 22]}

The spring-loaded jet injector uses is the spring mechanism that is drawn back. A trigger is then hit which release the spring creating a "jet stream" of vaccine or drug through the dermal layers of the skin. It is capable of subcutaneous, intramuscular or transdermal delivery. Each time the spring-load is activated the spring must then be manually redrawn to dose the next animal.

\section{Battery-powered jet injector $[21,22]$}

The battery-powered jet injector uses a small rechargeable battery pack to retract the dosing device. The dosing device has an electrical piston that is automatically redrawn after dosing. It is good for continuous use and minimizes worker fatigue. It is released by a small trigger. The injector resembles a battery powered hand drill. The battery-powered system administers subcutaneous, intramuscular or transdermal dosage depending on the recommended method.

\section{Gas-powered jet injector}

This type of injecting system was one of the first developed. It uses an gas or air cartridge attached to the gun either directly or indirectly through a tubing system to deliver power to the injector piston. When the trigger is activated it releases the piston and creates a jet stream of vaccine or drug subcutaneously, intramuscularly or Transdermally.

\section{Design of needle free injection [23]}

The air forced needle free injection systems are usually of three components which include an injection device, a disposable needle free syringe and an air cartridge. The injection device, the syringe is fabricated of a durable plastic. The syringe is sterilized prior since it is the only piece of the device that touches the skin. The syringe is fabricated such that, it should be disposed of after every use.

\section{Raw materials [23]}

These devices directly come in contact with the body, they must be fabricated from pharmacologically inert materials. The materials must be capable to endure high temperatures. They are heat sterilized. Air forced injection systems are available in different shapes and sizes. The external body of the device is made up of a thermoplastic which has characteristics such as high strength, lightweight. The example of such thermoplastic is polycarbonate. In order to make the polymer significant to mold, fillers are added. Due to the addition of fillers, the plastics become more durable, lightweight, and rigid. To modify the overall appearance, colorants are included into the plastic.

\section{The manufacturing process [23]}

There are many methods of producing each needle-free injection system. The following process focuses on the production of an airforced system. These systems are made of through a step by step procedure which involves molding the pieces, assembling them, and decorating and labeling the final product. The individual pieces are typically produced off-site and assembled by the needle free injection system manufacturer. All of the manufacturing is done under the sterile conditions to prevent the spread of disease.

\section{Making the pieces}

The first step requires is the production of the component plastic pieces from plastic pellets. The process is done by then the process is called as injection molding. Pellets of plastic are put into a large holding bin on an injection molding machine. They are heated to make them flowable.
The material is passed through a hydraulically controlled screw. As the screw rotates, the plastic is directed through a nozzle which then injects it into a mold. The mold is made up of two metal halves that form the shape of the part when brought together. When the plastic is in the mold, it is held under pressure for a specified amount of time and then allowed to cool. As it cools, the plastic inside hardens.

The mold pieces are separated and the plastic part falls out onto a conveyor. The mold then closes again and the process repeated. After the plastic parts are ejected from the mold, they are manually inspected to ensure that no significantly damaged parts are used.

\section{Assembling and labeling [24]}

The parts are next transported to an assembly line. In this production phase, various events occur. Machines apply markings that show dose levels and force measurements. These machines are specially calibrated so each printing is made precisely. Depending on the complexity of the device, human workers or machines may assemble the devices. This involves inserting the various pieces into the main housing and attaching any buttons.

\section{Packaging}

After the assembly step, the injection devices are put into packaging. They are first wrapped in sterile films and then put into cardboard or plastic boxes. Each part is packaged so movement is minimal to prevent damage. For consumer products, an instruction manual is included along with safety information. These boxes are then stacked on pallets and shipped via truck to distributors.

\section{Quality control [24]}

Quality control checks are done throughout the manufacturing process. Line inspectors check the plastic components to assure they conform to predetermined specifications. Visual inspections are the first test method, but measuring equipment is also used to check the dimensions including size and thickness. Instruments that can be used include laser micrometres, callipers and microscopes. Inspectors also check to make sure the printing and labeling is correct and that all the parts are included in the final packages. Since these devices can have various safety issues, their production is strictly controlled by the Food and Drug Administration (FDA). Each manufacturer must conform to various production standards and specifications.

\section{Mechanism of working [23]}

Needle-free injection technology is used force generated by a compressed gas (typically air, CO2 or nitrogen) to propel the vaccine at high velocity through a tiny orifice. When administered through the skin, an ultrafine stream of fluid penetrates the skin, delivering the vaccine in a fraction of a second to the skin, subcutaneous tissue, and intramuscular tissue. Injection event requires less than 0.5 seconds 22

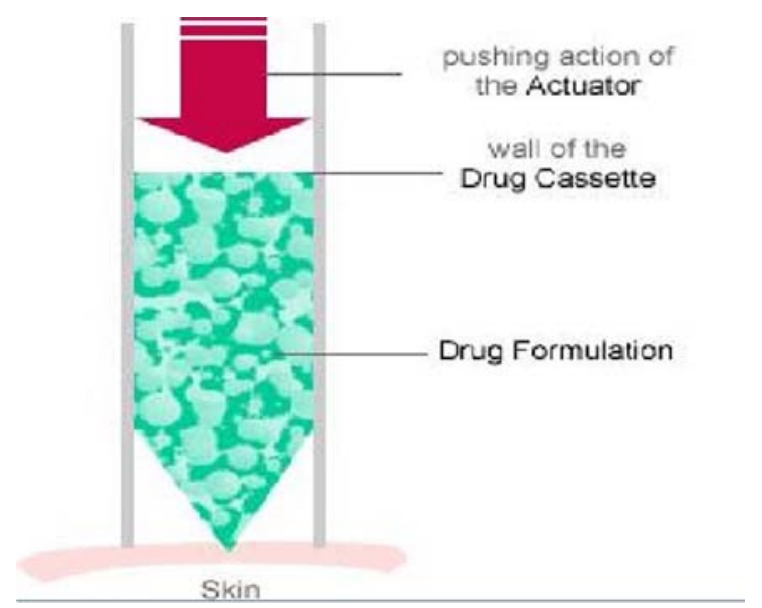

Fig. 4: Mechanism of working of needle free injection system [25] 


\section{Stages of delivery}

There are three stages in the needle free drug delivery:

- The peak pressure phase-is optimal pressure used to penetrate the skin is $<0.025 \mathrm{sec}$

- Delivery or dispersion phase is up to $0.2 \mathrm{sec}$

- Drop off phase is $<0.05 \mathrm{sec}$

The total amount of time required to deliver the vaccine is upto 0.5 seconds [26-29]

\section{Components of the needle free injection systems [1]}

\section{Nozzle}

The nozzle has the two significant functions it acts as the passage for the drug and as the surface which contacts the skin. The nozzle contains a flat surface and an orifice. The nozzle provides the surface which comes in contact in the skin and the orifice which has the drug passes through when injected. The orifice controls the drug stream diameter and speed. A stream diameter of approximately $100 \mu \mathrm{m}$ and traveling at $100 \mathrm{~m} / \mathrm{s}$ can achieve the desired injection depth of $2 \mathrm{~mm}$

\section{Drug reservoir}

The drug volume is holds the injection fluid inside the device.

\section{Pressure source}

The energy source is provided to the essential driving energy to the drug for injection. Then many devices in the market use either mechanical or stored pressure as energy storage elements. The mechanical method stores energy in a spring which is released by pushing a plunger to provide to the necessary pressure. The pressure storage method is used to compressed gas in a vessel which is released at the time of injection [26-29].

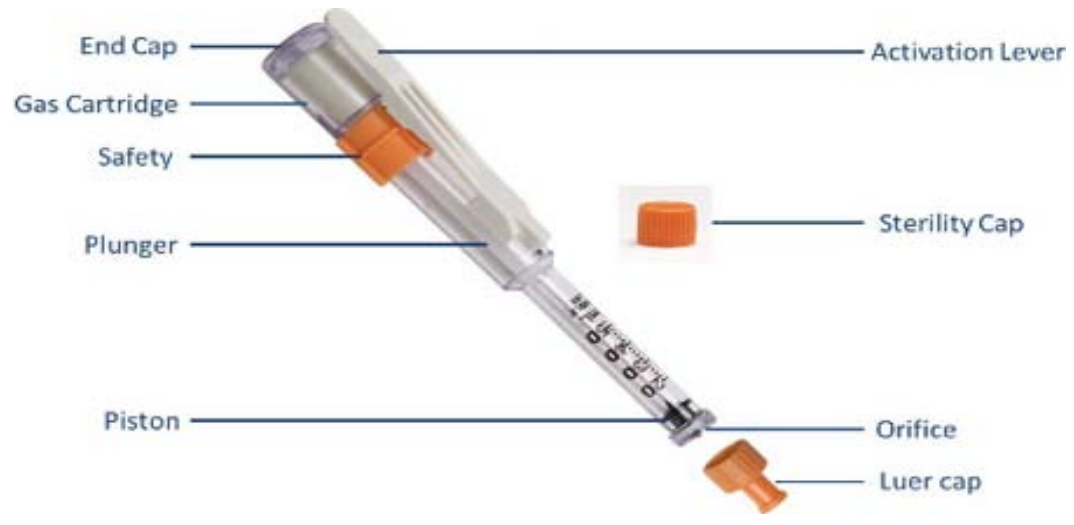

Fig. 5: Components of a needle-less injection device [30]

\section{Needle free injection technology}

Needle free injection technology encompasses a wide range of drug delivery systems that drive drugs through the skin using any of the forces as Lorentz, shock waves, pressure by gas or electrophoresis which propels the drug through the skin, virtually nullifying the use of a hypodermic needle. The devices are available in reusable forms. In contrast to the traditional syringes, neddle free injection technology does not only give the user freedom from unnecessary pain but drugs in the form of solid pallets can also be used administered. The future of this technology is promising ensuring virtually painless and highly efficient drug delivery.

The major drawback associated with this technology is the postadministration "wetness" of the skin which may, if not taken care of harbor dust and other untoward impurities. This technology is being backed by organizations of World Health Organization, Centers for
Disease Control and Prevention and various groups including the Bill and Melinda Gates Foundation. This technology is not only the touted to be beneficial for the pharma industry but also developing the world too find it highly useful in mass immunization programs, bypassing the chances of needle stick injuries and avoiding other complications including those arising due to the multiple uses of a single needle. Then the better patient compliance has been observed [31]

\section{Principle}

Neddle free injection technology harnesses energy stronger enough to propel a premeasured dose of a particular drug formulation, loaded in specific unique "cassettes" which can be rigged with the system. These forces may be generated from any of the ways ranging from high high-pressure fluids including gases, electromagnetic forces, shock waves or any other form of energy capable enough to impart motion to the medicament [32].

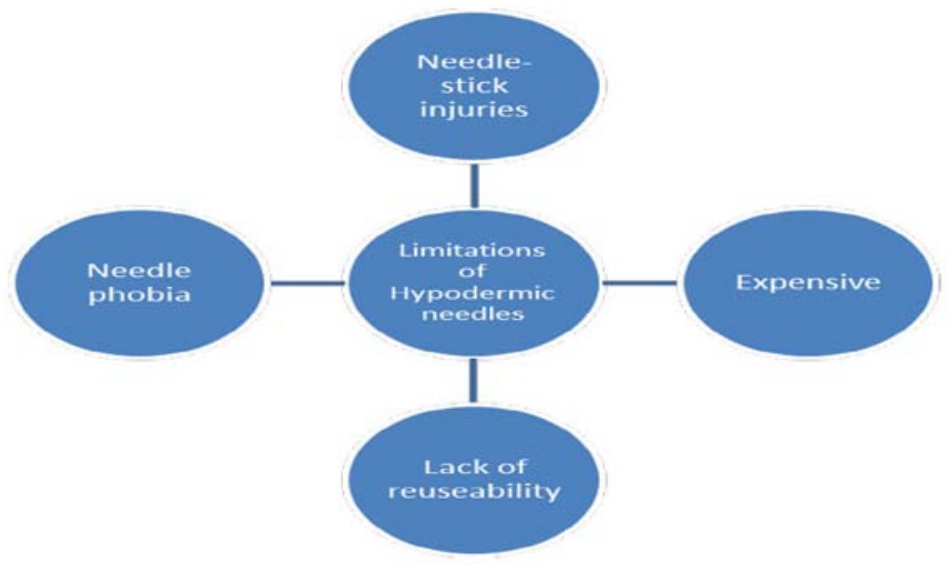

Fig. 6: Limitations of hypodermic needles 


\section{Classification of needle free injection technology [33-35]}

1. On the basis of working.

- Spring systems.

- Laser-powered.

- Energy propelled systems.

- Lorentz force.

- Gas propelled/air forced.

- Shockwaves.

2. On the basis of the type of load.

- Liquid.

- Powder.

- Projectile.

3. On the basis of the mechanism of drug delivery.

- Nano-patches.

- Sandpaper assisted delivery.

- Iontophoresis enabled.

- Micro-needles.

4. On the basis of site of delivery.

- Intradermal injectors.

- Intramuscular injectors.

- Subcutaneous injectors

\section{Mode of action of needle free injection technology}

\section{On the basis of working spring system}

Springs have been used to harbor energy and have been proven to be quite effective in powering neddle free injection technology devices. For neddle free injection technology, energy storage and further transmittance via spring is one of the easiest and simplest. However, the design of the spring must follow the standard protocols and the storage conditions must be simple or the spring will take a "set" over time deteriorating the performance of the device. The basic issue with respect to the design of the spring is that the force provided by the spring will reduce in proportion to the distance over which the load has been applied as according to the Hook's law. In simple words, in spring assisted neddle free injection technology, the pressure shall gradually decrease throughout the injection [36].

\section{Laser-powered}

A newer dimension of neddle free injection technology developed by Prof Jack Yoh and his team uses a laser-based system that blasts microscopic jets of drugs into the skin. The technology uses an erbiumdoped yttrium garnet laser (the one used in the care of laser resurfacing of the skin) to drive a very fine and precise stream of drug or medicament with the right amount of force. The laser is integrated with an adapter which holds the drug to be administered. The device also contains a chamber for water which is used to drive the medicine; however, the arrangement is so done that the drug is separated from the driving fluid (water) with the help of a membrane.

\section{Energy propelled system}

Commercial spring powered jet injectors offer little to no control over the pressure applied to the drug during the time of the injection; also these devices are often loud and sometimes painful. The force required to propel the drug so as to have a penetrating effect can also be generated by energy in various forms.

\section{Lorentz force [36]}

Researchers at MIT have engineered a neddle free injection technology device which uses Lorentz force to push a piston forward ejecting the drug at very high pressure and velocity (almost equal to that of sound in air). The main component of the device is the Lorentz force actuator which facilitates the entire process.

\section{Gas propelled/air forced}

Gas, as a power source, will be less suitable for reuse able devices unless special arrangement and design alterations or component modifications may be made such that the pressure is not lost, and the spring is reset for each injection, still, gas-powered neddle free injection technology have greater scope since compressed gas offer higher energy density than a metal spring. Gas-powered devices tend to be either single use or need a periodic replacement of the gas cartridge. Some devices employs gas as a simple spring where the stored gas accelerates the piston there are portable and compact, however, developing a gas spring which retain a specific proportion of the gas to work at the lapse of its shelf life is a major challenge.

To overcome such challenges, an alternate method has been developed which uses carbon dioxide liquefied at the storage temperature and pressure. This approach has been proven beneficial as a minimal loss of gas from the container inflicts virtually "no" or "zero" reduction in pressure. However, the pressure in such containers is highly sensitive to temperature with the pressure doubling between $0^{\circ}$ and $40^{\circ}$. This may affect the performance of the device if a broader operating temperature range is desired.

This problem can be sorted out by using a pressure regulator. Further research has led to the evolution of reusable, sophisticated and comparatively more portable gas-powered neddle free injection technology as in such systems (one developed by Team Consulting Ltd., Cambridge, UK) simple Butane combustion engine is used to power the device. The complete efficacy of this system is yet to be established, and data published. Major industries working for the development of neddle free injection technology systems have employed a technique of gas generation chemically in which the gas is produced at a reproducible and predictable rate to power the device. The reaction is initiated either mechanically or electrically, where the chemical "burns" generating gas [37].

The major drawbacks associated with this technology include:

- Complicated validation protocols.

- Foul odor due to combustion of reactants.

- Large volume manufacture of reactants.

\section{Shockwaves}

Shock waves are generated by any sudden release of energy. These disturbances carry energy and can be propagated through a medium. Researcher at the "Indian Institute of Science" (IISc) Bengaluru have developed a needless noninvasive drug delivery system employing this energy at supersonic levels. The prototype of this device consists of following major parts:

- Ignition system to ignite the "charge."

- Polymer tube which contains the explosive material which is suitably coated.

- Drug holding chamber to load the drug.

- The system also contains the cavity holder and metal foils [38].

\section{On the basis of the type of load}

\section{Liquid}

Liquid neddle free injection technology is the first variant of the neddle free injection technology systems and still, major players in the pharma industry are working on it. The entire mechanism of achieving a successful injection with a needle free system depends upon the ability of a liquid jet, stronger enough to penetrate the skin and the underlying fat layer without harming the skin or the integrity of the drug molecule. The mechanics involved in liquid neddle free injection technology is so complex that the recent studies have been carried out to understand the complete procedure of it [39]. 
Delivering fluid from neddle free injection technology involves a thorough application of fluid mechanics. The steps involved are:

- "Registration": The orifice of the device is placed exactly over the pores of the skin.

- Exact pressure: The fluid must be forced at an optimum pressure, stronger enough that it keeps the holes in the skin open and consistent enough that it avoids the resealing of the holes.

- Channel drilling: The initial pulse of the fluid drill a channel into the fat layer deep enough that the dose is drifted from the hole into the skin [40].

\section{Powder}

Powder needle free injection depends on being able to formulate the particles of sufficient density and accelerating them to sufficient velocity strong enough to penetrate the skin and in a quantity sufficient enough to reach the therapeutic dose levels [41]. This was made successful by using helium as a power source assisted by modifications in the ways of the formulation of the drug

Conversion of the drug either pure or along with excipients into hard particles of 10-50 nm in diameter, with a density approximately the same as a crystalline drug.

Coating the drug onto gold spheres which may act as a vector of few micrometres in diameter, this method is mostly applicable to DNA vaccines [42].

\section{Projectile/depot}

Highly advanced compared to the prior developed into this variant of the neddle free injection technology, the drug is processed into a long thin depot having sufficient mechanical strength strong enough to transmit a driving force to a pointed tip which may be formed either of an inert material or medicament itself. Generally, a depot is in the form of the cylinder measuring around $1 \mathrm{~mm}$ in diameter and few millimetres in length. This dimension may be small enough to limit the payload, but the quantity of the payload is sufficient enough for many new therapeutic proteins, antibodies, and other smaller molecules. The depot is strong enough to puncture the skin when punched with the sharp-tipped punch by applying a pressure of the order of 3-8 mega Pascal (MPa). For a depot preparation of around $1 \mathrm{~mm}$, only a few Newton's of force are required. The delivery device, therefore, would employ the transfer of energy from a suitable "spring" upon the depot [43].

\section{On the basis of the mechanism of drug delivery}

\section{Nano-patches}

The working of nano-patch or micro-projection depends on the use of an applicator to deliver the drug through the skin. Nano-patch projections are invisible to the naked eye and thereof are not anticipated to inflict fear into the people. Drug delivery using nanopatches have been highly efficient with respect to vaccines. Nanopatches enable the vaccine to reach the key immune cells located below the skin surface while the entire process is pain-free.

\section{Sandpaper assisted delivery}

Mostly, a 220 grit "sandpaper" kind of agent is rubbed onto the skin the skin so as to result in micro-derma abrasion phenomena where the superficial layer of the skin is removed, thereby facilitating the entire drug delivery process. Microdermabrasion has been widely accepted for cosmetic purposes. Sandpaper aided drug delivery has been successful in increasing the skin permeability, for several vaccines and other methods of Microdermabrasion have been used to facilitate the movement of drugs such as lidocaine, 5-flurouracil. Till now, vaccinations for traveller's diarrhoea and influenza have been developed using this technique (Clinical trials in progress) [44]

\section{Iontophoresis enabled}

The lipophilic nature of skin debars several salts and other molecules from entering the skin. By iontophoresis, a small electric current of about $0.5 \mathrm{~mA} / \mathrm{cm} 2$ is used to force several drug molecules across the skin. The working of this method involves the use of two electrodes as patches, where one acts as a drug reservoir, which can either be positively or negatively charged depending upon the nature of the drug, another patch is placed somewhere else on the body to complete the circuit [45].

\section{Micro-needle}

Micro-needle patches, as the name suggest, employs the use of thousands of tiny spikes all around $750 \mu$ long. These patches are pressed onto a person's skin while the spikes pierce the outer most layer of the skin so as to deliver the drug, while the piercing is not deep enough to hit the blood vessels or even the pain receptors so as to cause pain. Different types of micro-needles have been developed from the sophisticated metallic to plastic ones. While some are just "coated" with the drug, others are hollow having a liquid vaccine or the formulation filled inside. Micro-needle patches have not only proven to be highly effective but have even shown better patient compliance. However, certain limitations are associated with the use of micro-needle patches [46].

- Larger doses require bigger patch size.

- The formulation must be able to "coat" or "stick" on to the spikes on needle surface.

- In cases, if the needle itself is made of the drug, the formulation must have required the physicochemical property to maintain a sharp tip for adequate skin penetration.

- The depth of penetration of the micro-needle may differ from person to person, based on thickness, the toughness of the skin and reproducibility of the application.

- Movements of the body or the body part upon which the patch is applied may lead to dislodging of the needle [47].

\section{On the basis of site of delivery}

\section{Intradermal injector}

These systems have been employed to deliver comparatively newer, DNA-based vaccines to the intradermal layer. The system delivers the drug at a very shallow depth that is, between the layer of the skin [48].

\section{Intramuscular injector}

One of the most developed neddle free injection technology systems employed for intramuscular drug administration. Drug delivery via this system is the deepest among all. Drug delivery through neddle free injection technology devices has been most successful for vaccination [49].

\section{Subcutaneous injector}

Certain therapeutic proteins including the human growth hormones have been administered by this system. The medicament is delivered to the adipose layer just below the skin [49].

\section{CONCLUSION}

Needle free technology offers the very apparent benefit of minimizing patients fear regarding the use of a needle. Other benefits comprise very fast injection as compared to traditional needles and needle disposal issues are rarely seen. Not only it can assist the pharmaceutical industry in rising product sales, but also it has the extra potential to increase conformity with dosage regimens and enhanced outcomes. In the developing world, there are foremost challenges of disease transmission due to reuse of needles which can be overcome by the use of Needle free injection technology.

\section{AUTHORS CONTRIBUTIONS}

All the author have contributed equally

\section{CONFLICT OF INTERESTS}

\section{Declared none}

\section{REFERENCES}

1. Evolutionary approaches in the development of needle free injection technologies by Tarun Garg. Int J Pharm Pharm Sci 2012;4:590-6. 
2. Needle free injection technology: a review. Vishnu P, Sandhya M, Sreesh Kiran R, VaniCh V, Naveen Babu K. Int J Pharm 2012;2:148-55.

3. Lockhart MUS. Patent No. 69,199; 1936.

4. Transdermal and Topical Drug Delivery (ISBN: 0853694893) Pharmaceutical Press; 2003.

5. Gawkrodger DJ. Dermatology, an Illustrated Color Text. 3rd Ed. Edinburgh: Churchill Livingstone; 2002.

6. Bisset DL. Anatomy and biochemistry of the skin. In: kydonieus AF, Berner B. eds. Transdermal delivery of drugs. Vol. 1. Boca Raton: CRC Press; 1987. p. 29-42.

7. Buxton PK. ABC of dermatology. 3rd edition. London: BMJ Publishing Group; 1998.

8. C Scanlon Daniels. Needle-Free Injection: Pros and Cons. Available from: URL: http://www.highplainsdairy.org/ 2010/9_Daniels_Needle\%20Free\%20Injection_FINAL.pdf. [Last accessed on 15 Jan 2014]

9. http://www.pharmatutor.org/articles/needle-freeinjectiontechnology?page $=0,1$. [Last accessed on 15 Jan 2014]

10. Anubhav Arora, Murk R, Prausnitz, Samir Mitragotri. Microscale devices for transdermal drug delivery. Int J Pharm 2008;364:227-36

11. M Sunitha Reddy, M Ranjith Kumar, K Sanjay Kumar, Anil Goli P. Santhosh Kumar. Review on needle free drug delivery systems. Int J Rev Life Sci 2011;1:76-82.

12. Smita Kolhe, Sneha Sontakke. A review on needle free drug delivery system. Int J Curr Pharm Res 2013;2:30-6.

13. Weston Medical. Weston Medical. com FAQs. Available from: www.westonmedical.com/faqs.htm. [Last accessed on $20 \mathrm{Dec}$ 2017].

14. www.penjet.com. [Last accessed on 20 Dec 2017]

15. www.bioject.com. [Last accessed on 20 Dec 2017]

16. www.advantaget.com. [Last accessed on 20 Dec 2017]

17. www.equidyne.com. [Last accessed on 20 Dec 2017]

18. www.medajetxl.com. [Last accessed on 20 Dec 2017]

19. www.cdc.gov/nip/dev/N3draft0007doc. [Last accessed on 20 Dec 2017].

20. www.cdc.gov/nip/dev/N2draft000603doc. [Last accessed on 20 Dec 2017]

21. Houser TA, Sebranek JG, Bass TJ, Thacker BJ, Nilubol D, Thacker EL. Feasibility of transdermal, needleless injections for prevention of pork carcass defects. Meat Sci 2004;68:329-32.

22. PQA Plus. Available from: http://www.pork.org/filelibrary/ PQAPlus/PQAPlusEdBook.pdf. [Last accessed on 21 Sep 2011]

23. http://www.pharmatutor.org/articles/needle-freeinjectiontechnology?page $=0,1$ [Last accessed on 21 Sep 2011]

24. Mitragotri S. Current status and future prospects of needle-free liquid jet injectors. Nat Rev Drug Discovery 2006;5:543-8.

25. Needle free insulin devices-how does it work? $\mathrm{Br}$ J Diabetes Vascular Disease: Sherborne Gibbs Ltd; 2004. p. 4.

26. Needle free drug delivery technology by Dr. Roger G Harrison. Drug Delivery and Formulations, Innovations in pharmaceutical technology; 2004. p. 60-3.

27. Advances in disposable needle-free injector technology. Adam Levy, Weston Medical Ltd, Drug Delivery, Innovations in Pharmaceutical Technology; 1853. p. 100-9.

28. Pain-Free. Blunt-needle injection technology. Peter Crocker Kevin Maynard, Mervyn Little. Imprint Pharmaceuticals Ltd, Drug Delivery, Innovations in Pharmaceutical Technology; 1999. p. 111-5.

29. Needle free drug delivery systems: a review by Chandan Mohanty. Int J Pharm Res Dev 2011;3:7-15.
30. http://www.jtip.com/product_overview.html. [Last accessed on 15 Jan 2014]

31. Vishnu P, Sandhya M, Sreesh Kiran R, VaniCh V, Naveen Babu K. Needle free injection technology: a review. Int J Pharm 2012;2:148-55.

32. Ren T, Wang X, Yang PH. Vaccine and Needle free vaccination delivery system. J Microb Biochem Technol 2014;6:359-60.

33. Bioject Needle Free Injection Technology, Bioject Medical Technologies Inc., Leader in the Development of Needle-Free Injection Therapies. Available from: http://www.bioject.com/ technology. [Last accessed on 20 Dec 2017]

34. Zogenix.com, where Medicines Meet Technology. Available from: http://www.zogenix.com/content/technology/ dosepro. Htm.

35. King T. Intraject management forum conference on "NeedleFree Injection Systems and Auto-Injectors; 2015

36. Hunter I, George N. Vaccines and injections soon to be delivered via small needleless high-pressure jets. J Med Eng Phys; 2012.

37. http://therefusers.com/refusers-newsroom/vaccines-to-bedelivered-via-small-needleless-high-pressurejetspreventdisease-com. [Last accessed on 20 Dec 2017]

38. King T. Drug delivery: Needle-free systems. In: Encyclopedia of Pharmaceutical Technology. $3^{\text {rd }}$ ed. Vol. I. James Swarbrick Informa Healthcare; 2007. p. 1212.

39. Jagadeesh G, Prakash GD, Rakesh SG. Needleless vaccine delivery using micro-shock waves. Clin. Vaccine Immunol 2011;18:539-45.

40. Baker AB, Sanders JE. Fluid mechanics analysis of a springloaded jet injector. IEEE Trans Biomed Eng 1999; 46:235-42.

41. Schramm J, Mitragotri S. Transdermal drug delivery by jet injectors: energetics of jet formation and penetration. Pharm Res 2002;19:1673-9.

42. Potter C. Caretek Medical Device. Management forum conference on needle-free injection systems and auto-injectors. Management Forum. London, England; 2004

43. Prausnitz MR, Langer R. Transdermal drug delivery. Nat Biotechnol 2008;26:1261-8.

44. Cygnus, Inc. Sankyo Pharma and Cygnus Announce FDA Approval for Pediatric Use of GlucoWatch G2 Biographer. Available from: http://www.cygn.com/press/082802.html. [Last accessed on 20 Dec 2017]

45. Sarah CP. Under the skin of intra-dermal vaccines, proceedings of the national academy of sciences. Vol. 110. Published; 2013. p. 10049-51.

46. Richard T. Skin delivery of biological, transdermal patches, microneedles and needle-free injections: manufacturing lines roll as concepts become products. Drug Delivery 2013. Available from: http://www.ondrugdelivery.com/publications/ [Last accessed on 20 Dec 2017]

47. Transdermal_Microneedles_NFI_March_2013/Transdermal_Mi croneedles_NFI_March_2013_Low_Res.Pdf. [Last accessed on 20 Dec 2017]

48. Bioject Needle-Free Injection Technology, Bioject Medical Technologies Inc., Leader in the Development of Needle-Free Injection Therapies. Available from: http://www.bioject.com/ technology. [Last accessed on 20 Dec 2017].

49. Zogenix.com, where Medicines Meet Technology. Available from: http://www.zogenix.com/content/technology/dosepro.htm [Last accessed on 20 Dec 2017]. 